\title{
Local Lattice Strain Measurement using Geometric Phase Analysis of Annular Dark Field Images from Scanning Transmission Electron Microscopy
}

\author{
Jayhoon Chung, ${ }^{*}$ Guoda Lian,* and Lew Rabenberg ** \\ * Texas Instruments, Dallas, TX 75243 \\ ** Texas Materials Institute, University of Texas at Austin, Austin, TX 78712
}

Strain engineering to improve device performance is now widespread in the Si-based device industry. As such, there is a need for a technique that measures the local lattice strain on a routine basis at the size scale of the device. This metrology should be sensitive to small strains and should be able to resolve the spatial variations of the strain across the device. It should be practical and reproducible to apply it on a routine basis. All techniques that map strains at the sub-100 nm scale rely on some form of transmission electron microscopy or diffraction. [1-4] Although the high angle annular dark field mode of a scanning transmission electron microscope (HAADF-STEM) is common for high-resolution imaging, geometric phase analysis (GPA) of HAADF-STEM has not been used extensively. HAADF-STEM imaging has two strong advantages compared to normal high-resolution transmission electron microscopy (HRTEM). One is that more reproducible images can be recorded from HAADF-STEM images because it is less sensitive to experimental parameters. The other is that thicker samples can be imaged in HAADF-STEM, which can reduce strain relaxation effects due to sample thinning. However, hysteresis in scanning is a critical defect in the application of GPA to HAADF-STEM images. This paper shows how GPA of high-resolution images acquired in the HAADF-STEM can map strains at levels of accuracy and reproducibility needed for strained silicon device development.

The largest potential source of error in strain measurement in HAADF-STEM is the beam shift between scan lines due to the lens hysteresis. That is, when the beam reaches the end of its scan across the specimen, it should fly back to the start. If it fails to arrive precisely at the start, the subsequent line will be displaced, $\Delta \mathrm{u}_{\mathrm{x}}(\mathrm{y})$, as shown in Fig. 1. This "fly-back" defect involves a relatively large beam displacement; it should be the largest defect at modest magnifications. But, since it is essentially 1-dimensional, its effects on strain measurements can be eliminated by choosing a scan direction that is parallel to a principal axis of strain in the specimen.

Fig. 2 shows GPA strain analyses of an HAADF-STEM image of a strained $\mathrm{Si}_{82} \mathrm{Ge}_{18}$ alloy film on Si specimen. The scan was aligned parallel to a principle strain in the specimen, and GPA analysis of Fig. 2(a) was accomplished as usual [5] with the unstrained Si substrate at the bottom of Fig. 2(a) as reference. Fig. 2(b) is the $\varepsilon_{y y}$ strain map obtained by GPA analysis of Fig. 2(a); it shows no signs of the beam shifts between scan lines, even though the image spanned a $192 \mathrm{~nm} \times 192 \mathrm{~nm}$ field of view. It is another critical requirement of strain metrology to create 2-dimensional strain maps that span entire transistors, including source, channel, drain, and substrate. Mismatch strain measured from the dotted box in Fig. 2(b) is $1.21 \% \pm 0.41 \%$; this is in good agreement with a strain of $1.29 \%$ expected from continuum elasticity.

We have shown that Geometric Phase Analysis of High Angle Annular Dark Field -Scanning Transmission Electron Microscope images can be an effective, reproducible technique to measure local strains in the current node of Si based transistors. 


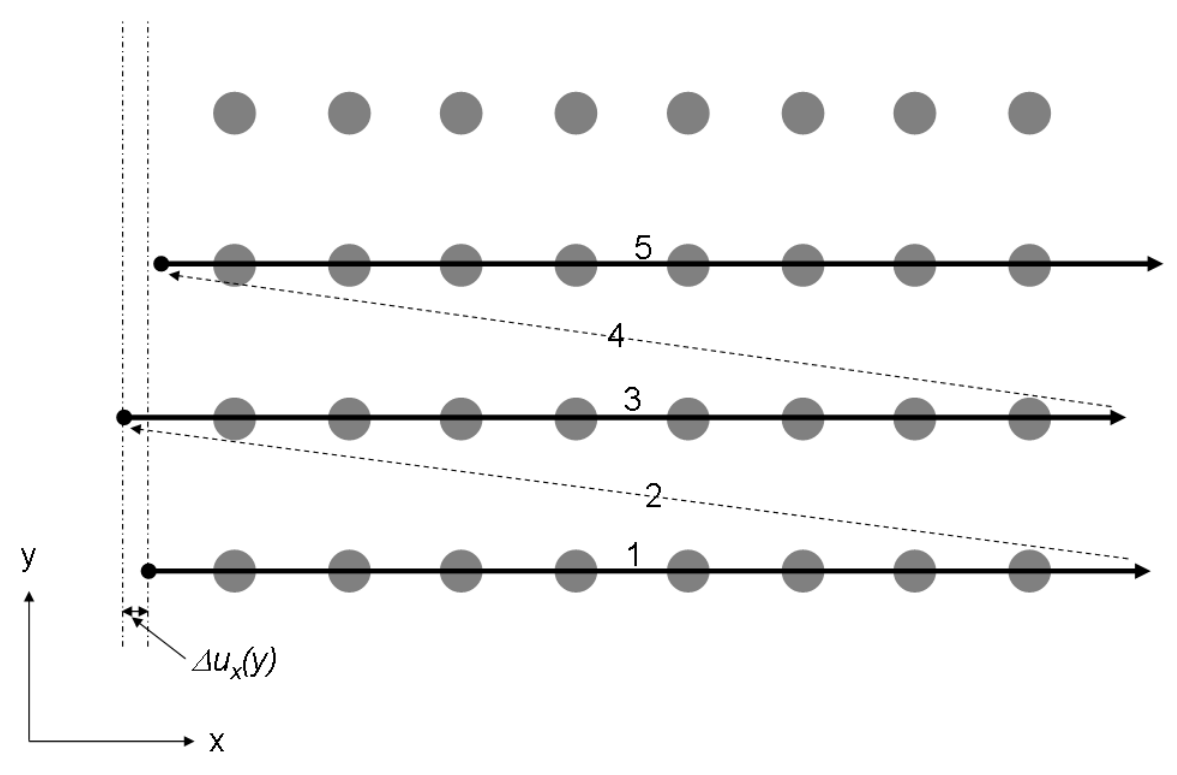

Fig. 1. Schematics of possible "fly-back" defect in scanning mode for HAADF-STEM imaging

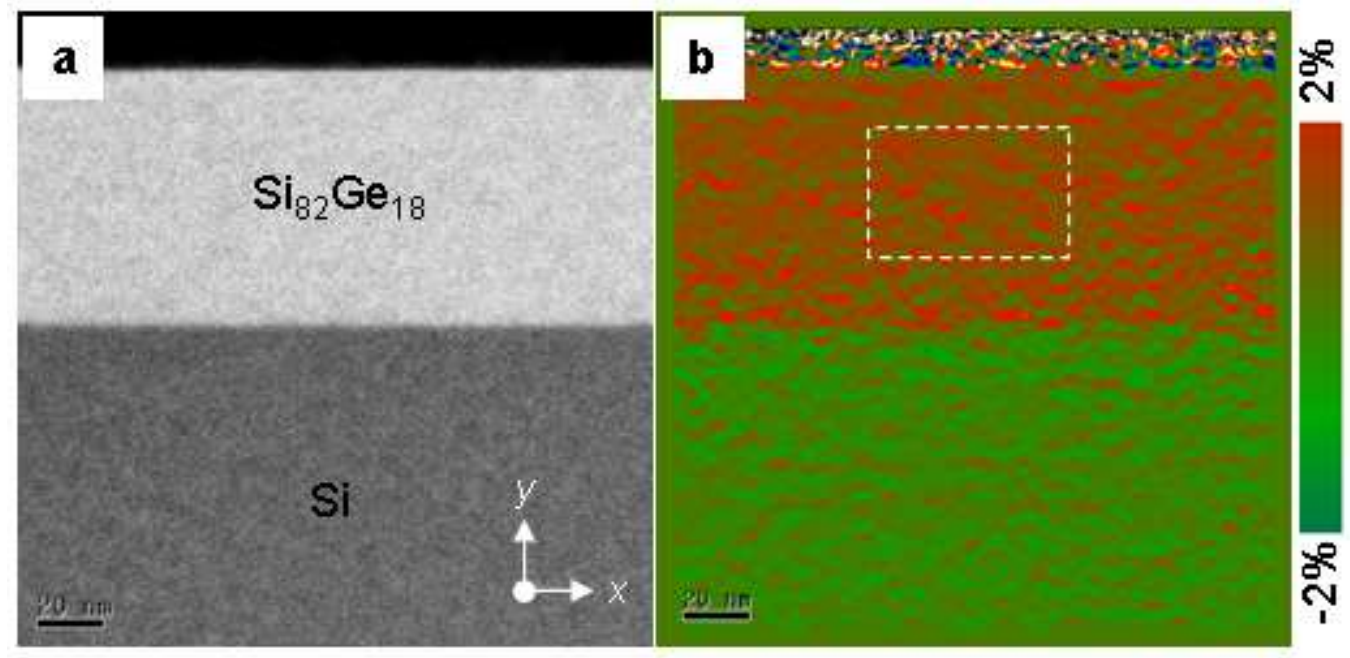

Fig. 2. (a) High-resolution HAADF-STEM image taken with the scan direction perpendicular to the interface between a $\mathrm{Si}$ single crystal substrate and a $\mathrm{Si}_{82} \mathrm{Ge}_{18}$ film. (b) $\varepsilon_{\mathrm{yy}}$ uniaxial strain map derived from (a) using GPA

\section{References}

[1] J. Huang, M. J. Kim, P. R. Chidambaram et al., Appl. Phys. Lett. 89 (2006) 063114.

[2] C. B. Vartuli, K. Jarausch, H. Inada et al., Microsc. Microanal. 13(suppl2) (2007) 836.

[3] M. Hÿtch, F. Houdellier, F. Hüe et al., Nature 453 (2008) 1086.

[4] J. Chung, G. Lian, and L. Rabenberg, Appl. Phys. Lett. 93 (2008) 081909.

[5] M. J. Hÿtch, E. Snoeck, and R. Kilaas, Ultramicroscopy 74 (1998) 131. 